\title{
Recent developments in the spermatogonial stem cell field
}

\author{
Dirk G. de Rooij ${ }^{1}$ \\ Reproductive Biology Group, Division of Developmental Biology, Department of Biology, Faculty of Science, \\ Utrecht University, Utrecht; Center for Reproductive Medicine, Academic Medical Center, University of Amsterdam, \\ Amsterdam, The Netherlands.
}

\begin{abstract}
This review aims at putting in perspective the many new developments in our understanding of spermatogonial multiplication and stem cell renewal in non-primate mammals.. In the rodent seminiferous epithelium, the spermatogonial compartment can be subdivided into $\mathrm{A}$, In and $\mathrm{B}$ spermatogonia, that show no, some or abundant nuclear heterochromatin, respectively. At first, it was thought that all A spermatogonia were spermatogonial stem cells while In and $\mathrm{B}$ spermatogonia were in the differentiation pathway. Then there appeared to be a class of so-called undifferentiated A spermatogonia, subdivided according to their topographical arrangement in to singles $\left(A_{s}\right)$, pairs $\left(A_{p r}\right)$ and chains of 4,8 and $16 A_{a l}$ spermatogonia. Four (in mouse and rat) subsequent generations of $\mathrm{A}$ spermatogonia together with In and B spermatogonia were called differentiating type spermatogonia. A socalled $A_{s}$ model was proposed in which the $A_{s}$ spermatogonia are the stem cells that self-renew by forming new singles or give rise to $A_{p r}$ spermatogonia that eventually will become spermatozoa. The $\mathrm{A}_{\mathrm{s}}$ model was challenged by the fragmentation model in which stem cell renewal was supposed to occur by way of fragmentation of clones of $\mathrm{A}_{\mathrm{al}}$ spermatogonia. However, the fragmentation model has not been supported by convincing evidence. Recently, it was shown that the compartment of $\mathrm{A}_{\mathrm{s}}$ spermatogonia can be subdivided into a class of the most primitive spermatogonial stem cells $(\mathrm{SSCu})$ that are able to form repopulating colonies after transplantation and transient stem cells ( $\mathrm{SSCt}$ ) that are not transplantable but still have some self-renewal capacity. The $\mathrm{SSCu}$ can be distinguished by the expression of the marker proteins ID4, PAX7 and BMI1. LIN28a and FXBW7 likely are negative markers. Interestingly, there is some evidence that the $\mathrm{SSCu}$ are resistant to the toxic effects of busulfan and irradiation. The most recent scheme of spermatogonial multiplication and stem cell renewal is discussed.
\end{abstract}

Keywords: BMI1, ID4, PAX7, germ cells, spermatogonial stem cells, spermatogenesis, SSC.

\section{Introduction}

Spermatogonial stem cells (SSCs) are at the beginning of the spermatogenic lineage. Spermatogenesis starts with a series of spermatogonial divisions through which large numbers of spermatogonia are produced that eventually divide into spermatocytes. Spermatocytes carry out the meiotic process and through the two meiotic divisions form haploid spermatids that develop into spermatozoa that leave the testis through the lumen of the seminiferous tubules. The whole spermatogenic process is tightly coordinated into the cycle of the seminiferous epithelium that in most species can be subdivided into 12 stages (Russell et al., 1990).

The compartment of spermatogonia has been extensively studied by many researchers (reviews: de Rooij and Russell, 2000; de Rooij and Griswold, 2012). Nevertheless, the question about the identity of the very first type of spermatogonia in the spermatogenic lineage, the spermatogonial stem cells, has not yet been decisively answered. This review depicts the evolution of our understanding of spermatogonial multiplication and stem cell renewal in non-primate mammals and finishes with the most recent model.

\section{Spermatogonial multiplication and stem cell renewal}

\section{The A0/A1-A4 model}

In the rat testis, Clermont described the presence of a sequential series of spermatogonial generations that were called A1, A2, A3, A4, In and B spermatogonia (Clermont, 1962). According to his definition of spermatogonial cell types, that has been widely accepted and is still in use to date, there are A spermatogonia that do not display any heterochromatin in their nuclei, In spermatogonia that do show some heterochromatin and B spermatogonia the nuclei of which show an abundant amount of heterochromatin. Clermont proposed that the A spermatogonia are the spermatogonial stem cells and that the A4 spermatogonia divide into both new A1 spermatogonia, that will start to produce spermatogonia for the next epithelial cycle, and In spermatogonia that will develop into spermatozoa. In this model, all type A spermatogonia can be regarded as stem cells.

In 1968, Clermont and Bustos-Obregon introduced the use of whole-mounts of seminiferous tubules to study spermatogonial multiplication and stem cell renewal (Clermont and Bustos-Obregon, 1968). In comparison with studying testis sections, this was an important improvement, as in whole mounts always the whole of the nuclei could be observed giving more morphological clues to identify spermatogonial cell types. In addition, the topographical arrangement of the clones of spermatogonia over the tubule basal lamina could now be studied. The use of the wholemount technique, led to the distinction of so-called A0 
spermatogonia, that were proposed to be quiescent singles and pairs of A spermatogonia that would function as reserve stem cells (Clermont and BustosObregon, 1968; Clermont and Hermo, 1975; Bartmanska and Clermont, 1983; Fig. 1).
The pioneering work by Clermont and coworkers has since been followed by extensive studies by many other groups and this has led to alternative models for spermatogonial multiplication and stem cell renewal better suiting the new data that emerged with time.

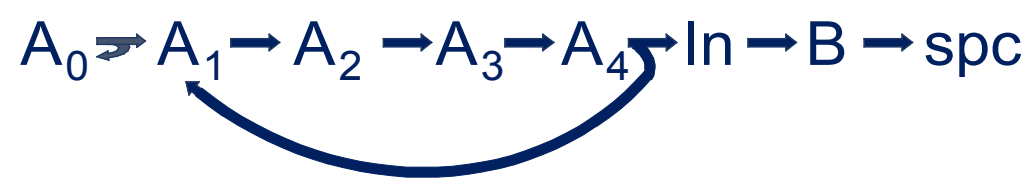

Figure 1. The $\mathrm{A}_{0} / \mathrm{A} 1-\mathrm{A} 4$ model. One of the earliest models for spermatogonial stem cell renewal in rodents. The $\mathrm{A}_{0}$ spermatogonia were supposed to be reserve stem cells that would only become active after cell loss. In this model spermatogonial differentiation starts with the In spermatogonia, while all A spermatogonia have stem cell capacities. From Clermont, 1962; Clermont and Bustos-Obregon, 1968; Clermont and Hermo, 1975; Bartmanska and Clermont, 1983. A0, A1, A2, A3, A4, In and B - Subsequent types of spermatogonia whereby In is short for Intermediate; spc - spermatocytes.

The $A_{s}$ model

In 1971, Huckins, studying the topographical distribution of spermatogonia in whole mounts of rat seminiferous tubules, proposed a different scheme for spermatogonial stem cell renewal. She called the A1 to B spermatogonia "differentiating" type spermatogonia and proposed the presence of a category of so-called "undifferentiated" spermatogonia earlier in the spermatogenic lineage. According to their topographical arrangement, these undifferentiated spermatogonia could be subdivided into single cells, pairs and chains of 4,8 or 16 cells, called $A_{\text {single }}\left(A_{s}\right), A_{\text {paired }}\left(A_{\text {pr }}\right)$ and $A_{\text {aligned }}\left(A_{a l}\right)$ spermatogonia, respectively (Huckins, 1971). The $A_{s}$ and $\mathrm{A}_{\mathrm{pr}}$ spermatogonia likely represented the $\mathrm{A}_{0}$ spermatogonia distinguished by Clermont and co- workers, who considered the clones of $\mathrm{A}_{\mathrm{al}}$ spermatogonia to be out of phase A1 to A4 spermatogonia. These ideas were endorsed by Oakberg (1971), working on mouse testis sections. Huckins and Oakberg proposed that the single $A_{s}$ spermatogonia are the stem cells of spermatogenesis. When these cells divide, the daughter cells can migrate away from each other or form a clone of 2 cells connected by an intercellular bridge. In the first case there is self-renewal as two new $\mathrm{A}_{\mathrm{s}}$ spermatogonia are formed and in the latter case it is the first step towards differentiation as the pairs will divide further to form clones of 4, 8 and 16 cells that in epithelial stage VIII will differentiate into A1 spermatogonia (Fig. 2; Huckins, 1971; Oakberg, 1971). This model was confirmed and/or expanded for mouse, Chinese hamster and ram (de Rooij, 1973; Lok et al., 1982).

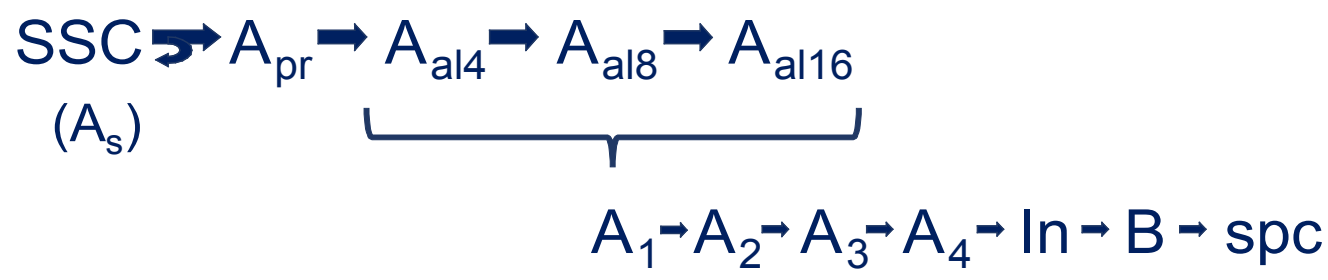

Figure 2. The $A_{s}$ model. This model is based on the presence of two categories of spermatogonia, undifferentiated and differentiating spermatogonia. The undifferentiated spermatogonia consist of singles $\left(A_{s}\right)$, pairs $\left(A_{p r}\right)$ and chains of 4,8 or 16 cells $\left(A_{a l}\right)$. The single cells are supposed to be the spermatogonial stem cells that produce new stem cells and Apr spermatogonia that will differentiate and eventually become spermatozoa. From Huckins, 1971; Oakberg, 1971; de Rooij, 1973.

The clones of $A_{s}, A_{p r}$ and $A_{a l}$ spermatogonia are not randomly distributed

In 2001, Chiarini-Garcia et al., using special fixation, staining and embedding techniques to distinguish $\mathrm{A}_{\mathrm{s}, \mathrm{pr}, \mathrm{al}}$ spermatogonia in sections, discovered that in the mouse the clones of $\mathrm{A}_{\mathrm{s}, \mathrm{pr}, \mathrm{al}}$ spermatogonia are not randomly distributed over the basal lamina. $\mathrm{A}_{\mathrm{s}, \mathrm{pr} \text {,al }}$ spermatogonia appeared to be preferentially localized in those areas of the tubule basal lamina opposing interstitial tissue and much less in areas where two tubules neighbored on each other (Chiarini-Garcia et al.,
2001). A similar phenomenon was observed in the rat (Chiarini-Garcia et al., 2003). This finding was beautifully confirmed and refined in life-imaging experiments in which the $A_{s, p r a l}$ spermatogonia where fluorescently labeled (Yoshida et al., 2007). The clones of $A_{s, p r, a l}$ spermatogonia were found to be localized in those areas of the tubule basal lamina that opposed arterioles and venules in the interstitial tissue. The existence of such areas in which the great majority of the $A_{\mathrm{s}, \mathrm{pr}, \mathrm{al}}$ spermatogonia reside indicates that this is the place where these cells are formed and therefore must include SSCs. These SSCs both maintain themselves by 
way of self-renewing divisions as well as produce spermatogonia that will develop into spermatozoa.

A computer model has been developed of such a niche in which the $A_{s}$ spermatogonia are localized in the middle of the niche, i.e. directly opposed to the interstitial arterioles and venules. $\mathrm{A}_{\mathrm{s}}$ spermatogonia were supposed to migrate in random directions whereby virtually all $A_{s}$ that moved out of the niche formed $A_{p r}$ spermatogonia at their next division. Putting in parameters obtained during previous cell kinetic studies, it was shown that such a niche allows longterm steadystate spermatogenesis (de Rooij and van Beek, 2013).

\section{The clonal fragmentation model}

The Yoshida group has set up a life-imaging system that, for 3 to 4 days, makes it possible to follow the behavior of spermatogonia on the basal lamina of those seminiferous tubules that lie directly under the tunica albuginea of a mouse testis (Nakagawa et al.,
2007; Yoshida et al., 2007; Hara et al., 2014). The spermatogonia were fluorescently labeled by means of a tamoxifen-dependent Cre recombinase under the upstream sequence of the Ngn3 gene or the Gfral gene. Lineage tracing experiments were carried out and it appeared that some spermatogonia expressing the differentiation marker NGN3 could form longterm patches of spermatogenic cells. As these patches did include multiple generations of labeled spermatogenic cells, these patches must have been formed by stem cells. Furthermore, during the life-imaging experiments occasionally chains of $A_{p r}$ and $A_{a l}$ spermatogonia were seen to fragment into singles and pairs. A model was proposed in which $A_{p r}$ and $A_{a l}$ spermatogonia retained the capacity to dedifferentiate and became spermatogonial stem cells again through fragmentation of the clones of $\mathrm{A}_{\mathrm{al}}$ spermatogonia into smaller clones of one or two cells (Fig. 3). A computer model was developed that fitted with these data (Nakagawa et al., 2007; Klein et al., 2010; Nakagawa et al., 2010; Hara et al., 2014).

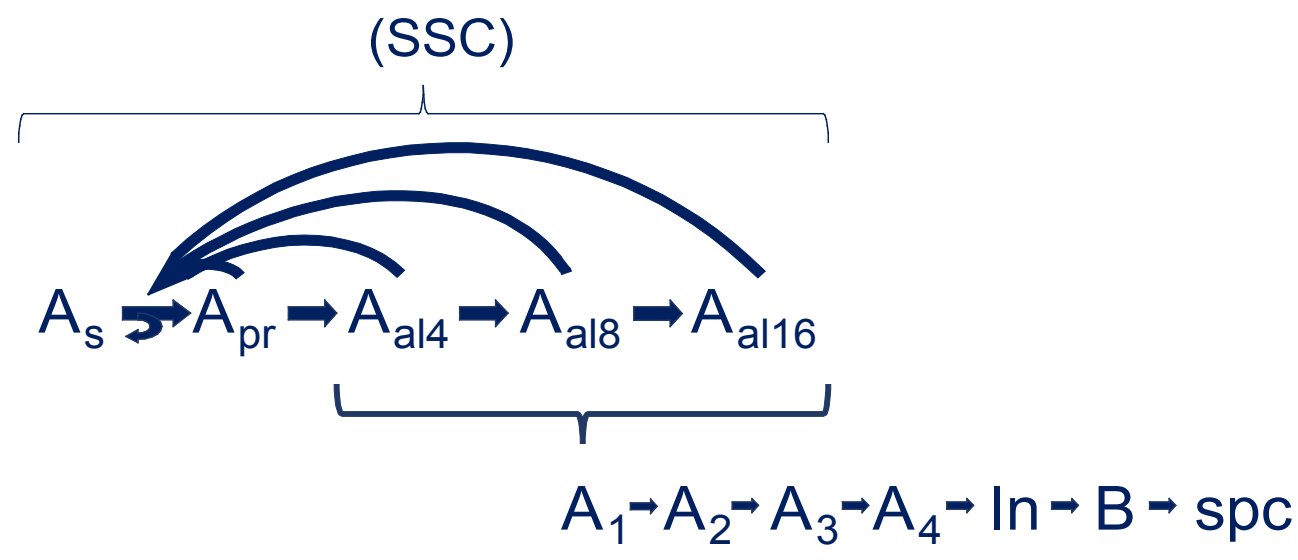

Figure 3. The fragmentation model. In this model $A_{p r}$ and $A_{a l}$ spermatogonia are supposed to be potential stem cells as in a life-imaging set up the pairs and chains occasionally fragment into smaller clones including single cells. In addition, lineage tracing experiments after labeling with the differentiation associated gene ngn 3 , show the formation of longterm patches of multi generations of spermatogenic cells, suggesting the occurrence of dedifferentiation of spermatogonia early in the differentiation pathway. From Nakagawa et al., 2007; Hara et al., 2014).

The authors also counted the number and size of the patches formed by NGN3 positive stem cells. At 3 months after labeling, they estimated the average length of the patches to be $1.4 \mathrm{~mm}$. As total tubule length was found to be $1700 \mathrm{~mm}$, the authors estimated that there were about 2000 actual SSCs per mouse testis (Nakagawa et al., 2007). This number is much different from the number of $35.000 \mathrm{~A}_{\mathrm{s}}$ spermatogonia per mouse testis estimated during cell counts (Tegelenbosch and de Rooij, 1993). However, the authors also found that for both NGN3 and GFRA1 marker proteins, both number and size of the patches very strongly depended on the time point after labeling at which these parameters were estimated. Especially during the first couple of months after labeling, the number of patches steeply decreased while their size increased. Therefore, estimation of the number of "actual" stem cells is strongly dependent on the timepoint after labeling at which the size of the patches is measured. Hence, the validity of determining the numbers of SSCs in lineage tracing experiments by the size of patches formed by labeled stem cells is highly questionable.

In a recent life-imaging experiment, it was established that the $A_{s}$ spermatogonia divided once every 10 days, the $A_{p r}$ once every 12 days and the $A_{a l}$ once every 13 days (Hara et al., 2014). These data suggested that the $\mathrm{A}_{\mathrm{s}, \mathrm{pr}, \mathrm{al}}$ spermatogonia divide considerably less than once every epithelial cycle which takes 8.6 days in the mouse (Oakberg, 1957). Unfortunately, this finding is incompatible with normal steady state kinetics in the seminiferous epithelium in rodents. During every epithelial cycle in the mouse, about $85 \%$ of the total number of $\mathrm{A}_{\mathrm{s}, \mathrm{pr}, \mathrm{al}}$ spermatogonia differentiates into A1 spermatogonia (Tegelenbosch and de Rooij, 1993) and in the Chinese hamster and the ram these figures are 80 and $70 \%$, respectively (Lok et al., 1982; Lok and de Rooij, 1983). It is mathematically impossible that the 15 to $35 \%$ of the $A_{\text {s,pr,al }}$ spermatogonia that do not differentiate into A1 spermatogonia can replenish the cells lost to differentiation by dividing less than once during the subsequent epithelial cycle. Therefore, the cell cycle 
data obtained in the life-imaging system cannot be correct. Indeed, data from cell counts and cell-kinetic data obtained through $3 \mathrm{H}$-thymidine labeling studies all indicated that on average $A_{\text {s,pr,al }}$ spermatogonia divided 2 to 3 times per epithelial cycle in mouse, Chinese hamster and ram (Lok et al., 1982; Lok and de Rooij, 1983; Lok et al., 1983; Tegelenbosch and de Rooij, 1993). Possibly, in the life-imaging situation there is photon-damage of the $\mathrm{A}_{\mathrm{s}, \mathrm{pr}, \mathrm{al}}$ spermatogonia in the observation area, which may also explain the fragmentation of larger clones. Alternatively, the spermatogonia in the area studied are not representative for the whole population of $\mathrm{A}_{\mathrm{s}, \mathrm{pr}, \mathrm{al}}$ spermatogonia in the testis. In support of the latter possibility, in the lifeimaging system only the surface of the seminiferous tubules directly under the tunica albuginea can be viewed while the arterioles and venules under which the $\mathrm{A}_{\mathrm{s} \text {,pral }}$ spermatogonial niche is situated, are lying somewhat deeper and to the edges of the tubule areas studied. Therefore, the plane of the $\mathrm{A}_{\mathrm{s} \text {,pr,al }}$ spermatogonial niche will in fact be perpendicular to the plane of the observation field. The niche area cannot be studied in the life-imaging set up but only the cells that spill out of that region. This may also explain why virtually all $A_{s}$ spermatogonia were seen to form $A_{p r}$ spermatogonia at division, these $\mathrm{A}_{\mathrm{s}}$ spermatogonia were outside of the niche and should indeed differentiate. Finally, it has not been proven that the single A spermatogonia formed by fragmentation of clones of 4 to 16 cells can indeed behave as stem cells. From epithelial stage II onwards the clones of 4 to 16 cells are already quiescent and competent to differentiate into A1 spermatogonia in response to an increased level of retinoic acid (Endo et al., 2015) and it does not seem likely they can dedifferentiate again to stem cells. Evidence should be provided that the single $A_{s}$ spermatogonia that were formed by clonal fragmentation were functional stem cells and did not differentiate into A1 spermatogonia.

Taken together, there is unsufficient basis for the clonal fragmentation model and this model is not compatible with longterm steady state kinetics of the spermatogenic process.

\section{Heterogeneity among the $A_{s}$ spermatogonia}

In contrast to the proposed expansion of the spermatogonial stem cell compartment from $A_{s}$ to $A_{p r}$ and $\mathrm{A}_{\mathrm{al}}$ spermatogonia in the clonal fragmentation model, recent findings indicate that even only few of the $\mathrm{A}_{\mathrm{s}}$ spermatogonia are stem cells. Pioneering work by the Oatley group indicates an important role for Inhibitor of differentiation 4 (ID4) in the spermatogonial stem cell compartment. This group established that ID4 is only expressed in a few $\mathrm{A}_{\mathrm{s}}$ spermatogonia and these cells are present throughout the stages of the epithelial cycle (Oatley et al., 2011). Furthermore, this group purified ID4 positive spermatogonia and by using the spermatogonial transplantation assay they established that only ID4 positive spermatogonia can form repopulating colonies in recipient mouse testes. They estimated the number of ID-4 positive spermatogonia to be about 6000 per testis (Chan et al., 2014). Intriguingly, the ID4 positive spermatogonia did not reside in the $A_{s, p r a l}$ spermatogonial niche, as they were specifically absent from areas of the tubule basal lamina bordering on interstitial arterioles and venules.

The notion that ID4 positive spermatogonia are stem cells was confirmed by studies by Sun et al. (2015). In lineage-tracing experiments these authors showed that ID4 positive spermatogonia could give rise to longterm patches containing multiple generations of spermatogenic cells, proving the stem cell capacity of these spermatogonia.

The results on ID4 of both groups thus indicate that ID4 is a marker for SSCs that are capable of repopulating a recipient mouse testis and to form longterm patches of spermatogenic cells. Importantly, not all $\mathrm{A}_{\mathrm{s}}$ spermatogonia in the testis have this capacity, only 6000 of the $35.000 \mathrm{~A}_{\mathrm{s}}$ present. The finding that ID4 positive $A_{s}$ spermatogonia are totally absent from the $A_{s, p r, a l}$ spermatogonial niche indicates that this a rather special type of $A_{s}$ spermatogonia that in this review will further be called "ultimate" stem cells ( $\mathrm{SSCu})$.

In addition to ID4, more candidates for $\mathrm{SSCu}$ specific genes/proteins have been described. First, there is paired box transcription factor 7 (pax7). PAX7 is expressed in a small subset of $A_{s}$ spermatogonia that in lineage tracing experiments were able to form longterm patches of spermatogenic cells (Aloisio et al., 2014). PAX7 positive cells were found to co-express ID4. Pax7 positive cells were even more rare than ID4 positive ones as about 400-500 were found in both the prepuberal and adult mouse testis. Furthermore, PAX7 positive cells are actively proliferating, over $40 \%$ of these cells had incorporated EdU at $1 \mathrm{hr}$ after administration. Apparently all PAX7 positive spermatogonia are in active cell cycle. Possibly, the PAX7 positive cells represent those ID4 positive cells that are proliferating.

Second, another recently discovered marker gene for $\mathrm{SSCu}$ is $\mathrm{B}$ cell-specific Moloney murine leukemia virus integration sitel (bmil; Komai et al., 2014). Similar to ID4 and PAX7, BMI1 is only expressed in $A_{s}$ spermatogonia and occasionally in $A_{p r}$ spermatogonia. BMI1 positive cells could also express ID4 and in lineage tracing experiments BMI1 positive cells were able to form longterm patches of multigenerations of spermatogenic cells indicating these cells have stem cell capacity. The number of BMI1 positive cells was estimated to be about 5000 per testis which is comparable to the number of ID4 positive cells (Chan et al., 2014).

Interestingly, PAX7 positive $\mathrm{SSCu}$ were found to be resistant to busulfan and BMI1 positive $\mathrm{SSCu}$ were more resistant to both busulfan and irradiation (Aloisio et al., 2014; Komai et al., 2014). This opens the possibility that the $\mathrm{SSCu}$ are the cells most resistant to toxic factors. This aspect will have to be studied in further detail.

Finally there are factors that may well play a role in the regulation of the behaviour of $\mathrm{SSCu}$ but whose expression does not seem restricted to a few $A_{s}$ spermatogonia as these proteins are also present in $A_{p r}$ 
spermatogonia. Nanos2 and RB deficiency causes a rather rapid depletion of the spermatogenic stem cell pool as evidenced by a complete loss of germ cells (Sada et al., 2009; Hu et al., 2013; Yang et al., 2015). As yet, it is not possible to predict the specific role of NANOS2 and RB in the regulation of $\mathrm{SSCu}$ behavior.

In view of the fact that the repopulation of a recipient mouse testis, with little or no endogenous spermatogenesis, depends on the transplantation of $\mathrm{SSCu}$, the gene F-box and WD-40 domain protein 7 (fbxw7) is of great interest. In cultured germ line stem (GS) cells, FBXW7 deficiency enhanced the number of colonies formed after transplantation by these cells and overexpression of FBXW7 decreased this number (Kanatsu-Shinohara et al., 2014). This finding indicates that $\mathrm{FXBW} 7$ functions at the regulation of the decision of $\mathrm{SSCu}$ to stay the most primitive type of SSC or to abandon this capacity and to proceed to a more differentiated type of cell. This suggests that FXBW7 is a negative marker for $\mathrm{SSCu}$.

Another possible negative marker for $\mathrm{SSCu}$ is Lin-28 homolog A, encoding for LIN28A, that was found to be expressed in $\mathrm{A}_{\mathrm{s}}$ through $\mathrm{A} 4$ spermatogonia (Zheng et al., 2009; Hu et al., 2010; Gaytan et al., 2013). LIN28A has a role in the outgrowth of the clones of $A_{p r}$ and $A_{a l}$ spermatogonia and deficiency for LIN28A does not affect the $\mathrm{SSCu}$ population (Chakraborty et al., 2014). A proportion of the $\mathrm{A}_{\mathrm{s}}$ spermatogonia does not express LIN28A and the authors suggest that these $A_{s}$ spermatogonia are stem cells while the LIN28A positive $A_{s}$ are progenitor cells (further called transient SSC or SSCt) predestined to at some point form $\mathrm{A}_{\mathrm{pr}}$ spermatogonia.

Taken together these data suggest that at the start of spermatogenesis there are SSCu spermatogonia that are in any case ID4 positive, not always PAX7 positive and likely BMI1 positive. As the expression of FBXW7 diminishes their capacity to repopulate a recipient mouse testis upon transplantation, SSCu likely do not or only weakly express FBXW7. In addition, LIN28A may also be a negative marker for SSCu. In view of these results, the present state of the art in this field suggest the phenotype of $\mathrm{SSCu}$ to be as depicted in Fig. 4A.

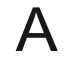

\section{Markers for SSCu}
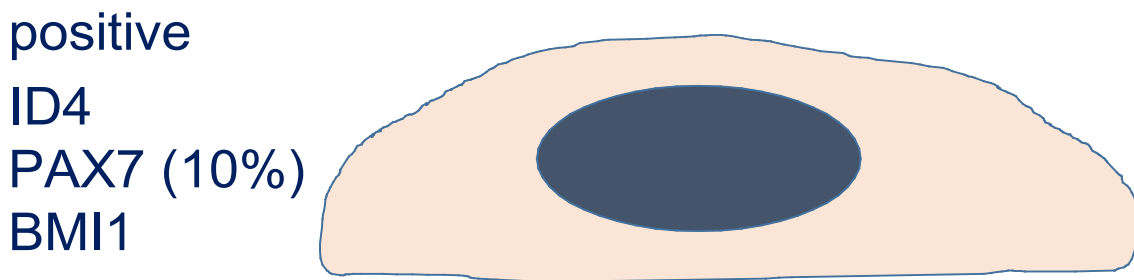

negative LIN28a FBXW7

$\mathrm{B}$

SSCu $\boldsymbol{\sim}$ SSCt $\boldsymbol{\sim} \mathrm{A}_{\mathrm{pr}} \rightarrow \mathrm{A}_{\mathrm{al} 4} \rightarrow \mathrm{A}_{\mathrm{al} 8} \rightarrow \mathrm{A}_{\mathrm{al} 16}$

$\left(A_{s}\right)$

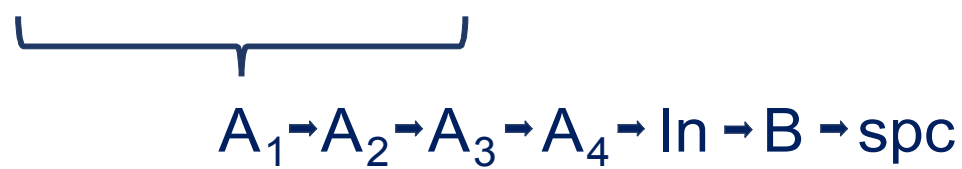

Figure 4. A. Recent data indicate that the compartment of $\mathrm{A}_{\mathrm{s}}$ spermatogonia can be subdivided into ultimate stem cells $(\mathrm{SSCu})$ and transient stem cells $(\mathrm{SSCt})$ that cannot fully maintain themselves. Various proteins have been found that within the spermatogenic lineage are specifically expressed in $\mathrm{SSCu}$ or among the undifferentiated spermatogonia specifically lack particular proteins. This figure depicts the present knowledge on the phenotype of SSCu. From Oatley et al., 2011; Aloisio et al., 2014; Chakraborty et al., 2014; Kanatsu-Shinohara et al., 2014; Komai et al., 2014; Sun et al., 2015. B. The dual stem cell model. This model takes into account the presence of a generation of most primitive stem cells, called $\mathrm{SSCu}$ and a subsequent generation of transient stem cells called SSCt. This model is in fact similar to the $A_{s}$ model except that the $A_{s}$ spermatogonia are subdivided into two subsequent categories. From Aloisio et al., 2014; Chan et al., 2014.

\section{Newest model for spermatogonial multiplication and stem cell renewal and remaining questions}

As already suggested by the group of Oatley and Aloisio et al, the present data indicate that there is a relatively small compartment of $\mathrm{SSCu}$ at the start of spermatogenesis (Aloisio et al., 2014; Chan et al., 2014). These cells are localized outside of the $A_{s, p r a l}$ spermatogonial niche and are the only cells that can repopulate a recipient mouse testis. However, because of their small number, the $\mathrm{SSCu}$ themselves cannot be responsible for the total of the cyclic production of A1 
spermatogonia. In addition, as many $\mathrm{A}_{\mathrm{s}}$ spermatogonia will divide into $A_{\text {pr }}$ each epithelial cycle, the few $\mathrm{SSCu}$ present will not be able to produce enough new $A_{s}$ to replenish the $A_{s}$ that divided into $A_{p r}$ during the previous epithelial cycle. Therefore, one has to accept that the $\mathrm{SSCu}$ give rise to $\mathrm{A}_{\mathrm{s}}$ spermatogonia that still have the capacity to renew themselves but not enough to maintain longterm steady state spermatogenesis. Probably, on average the percentage of self-renewing divisions of these $\mathrm{A}_{\mathrm{s}}$ is less than $50 \%$ and the deficit that is formed is replenished by divisions of $\mathrm{SSCu}$. In this situation the $\mathrm{A}_{\mathrm{s}}$ spermatogonia that are not $\mathrm{SSCu}$ are transient and can be called SSCt. These considerations than leads to the scheme of spermatogonial multiplication and stem cell renewal depicted in Fig. 4B, a notion that was already proposed by Oatley et al. and Aloisio et al. (Aloisio et al., 2014; Chan et al., 2014).

Many questions still remain. For example, the relationship between the cells expressing ID4, PAX7 and BMI1. Do they constitute one population of cells that express these markers at different stages of the epithelial cycle or their cell cycle? How do the $\mathrm{SSCu}$ differentiate into SSCt? Clearly, the discovery of the $\mathrm{SSCu}$ has been a real breakthrough in this field and our understanding of spermatogonial stem cells will greatly expand in the near future.

\section{References}

Aloisio GM, Nakada Y, Saatcioglu HD, Pena CG, Baker MD, Tarnawa ED, Mukherjee J, Manjunath H, Bugde A, Sengupta AL, Amatruda JF, Cuevas I, Hamra FK, Castrillon DH. 2014. PAX7 expression defines germline stem cells in the adult testis. J Clin Invest, 124:3929-3944.

Bartmanska J, Clermont Y. 1983. Renewal of type A spermatogonia in adult rats. Cell Tissue Kinet, 16:135143.

Chakraborty P, Buaas FW, Sharma M, Snyder E, de Rooij DG, Braun RE. 2014. LIN28A marks the spermatogonial progenitor population and regulates its cyclic expansion. Stem Cells, 32:860-873.

Chan F, Oatley MJ, Kaucher AV, Yang QE, Bieberich CJ, Shashikant CS, Oatley JM. 2014. Functional and molecular features of the Id4+ germline stem cell population in mouse testes. Genes Dev, 28:1351-1362.

Chiarini-Garcia H, Hornick JR, Griswold MD, Russell LD. 2001. Distribution of type A spermatogonia in the mouse is not random. Biol Reprod, 65:1179-1185.

Chiarini-Garcia H, Raymer AM, Russell LD. 2003. Non-random distribution of spermatogonia in rats: evidence of niches in the seminiferous tubules. Reproduction, 126:669-680.

Clermont Y. 1962. Quantitative analysis of spermatogenesis of the rat: a revised model for the renewal of spermatogonia. Am J Anat 111:111-129.

Clermont Y, Bustos-Obregon E. 1968. Reexamination of spermatogonial renewal in the rat by means of seminiferous tubules mounted "in toto". Am J
Anat, 122:237-247.

Clermont Y, Hermo L. 1975. Spermatogonial stem cells in the albino rat. Am J Anat, 142:159-175.

de Rooij DG. 1973. Spermatogonial stem cell renewal in the mouse. I. Normal situation. Cell Tissue Kinet, 6:281-287.

de Rooij DG, Russell LD. 2000. All you wanted to know about spermatogonia but were afraid to ask. $J$ Androl 21:776-798.

de Rooij DG, Griswold MD. 2012. Questions about spermatogonia posed and answered since 2000. J Androl, 33:1085-1095.

de Rooij DG, van Beek ME. 2013. Computer simulation of the rodent spermatogonial stem cell niche. Biol Reprod, 88:131.

Endo T, Romer KA, Anderson EL, Baltus AE, de Rooij DG, Page DC. 2015. Periodic retinoic acidSTRA8 signaling intersects with periodic germ-cell competencies to regulate spermatogenesis. Proc Natl Acad Sci USA, 112:E2347-2356.

Gaytan F, Sangiao-Alvarellos S, Manfredi-Lozano M, Garcia-Galiano D, Ruiz-Pino F, Romero-Ruiz A, Leon S, Morales C, Cordido F, Pinilla L, TenaSempere M. 2013. Distinct expression patterns predict differential roles of the miRNA-binding proteins, Lin28 and Lin28b, in the mouse testis: studies during postnatal development and in a model of hypogonadotropic hypogonadism. Endocrinology, 154:1321-1336.

Hara K, Nakagawa T, Enomoto H, Suzuki M, Yamamoto M, Simons BD, Yoshida S. 2014. Mouse spermatogenic stem cells continually interconvert between equipotent singly isolated and syncytial states. Cell Stem Cell 14:658-672.

Hu J, Wang F, Zhu X, Yuan Y, Ding M, Gao S. 2010. Mouse ZAR1-like (XM_359149) colocalizes with mRNA processing components and its dominantnegative mutant caused two-cell-stage embryonic arrest. Dev Dyn, 239:407-424.

Hu YC, de Rooij DG, Page DC. 2013. Tumor suppressor gene $\mathrm{Rb}$ is required for self-renewal of spermatogonial stem cells in mice. Proc Natl Acad Sci USA, 110:12685-12690.

Huckins C. 1971. The spermatogonial stem cell population in adult rats. I. Their morphology, proliferation and maturation. Anat Rec, 169:533-557.

Kanatsu-Shinohara M, Onoyama I, Nakayama KI, Shinohara T. 2014. Skp1-Cullin-F-box (SCF)-type ubiquitin ligase FBXW7 negatively regulates spermatogonial stem cell self-renewal. Proc Natl Acad Sci USA, 111:8826-8831.

Klein AM, Nakagawa T, Ichikawa R, Yoshida S, Simons BD. 2010. Mouse germ line stem cells undergo rapid and stochastic turnover. Cell Stem Cell, 7:214224.

Komai Y, Tanaka T, Tokuyama Y, Yanai H, Ohe S, Omachi T, Atsumi N, Yoshida N, Kumano K, Hisha H, Matsuda T, Ueno H. 2014. Bmil expression in long-term germ stem cells. Sci Rep, 4:6175.

Lok D, Weenk D, de Rooij DG. 1982. Morphology, proliferation, and differentiation of undifferentiated spermatogonia in the Chinese hamster and the ram. Anat Rec, 203:83-99. 
Lok D, de Rooij DG. 1983. Spermatogonial multiplication in the Chinese hamster. III. Labelling indices of undifferentiated spermatogonia throughout the cycle of the seminiferous epithelium. Cell Tissue Kinet, 16:31-40.

Lok D, Jansen MT, de Rooij DG. 1983. Spermatogonial multiplication in the Chinese hamster. II. Cell cycle properties of undifferentiated spermatogonia. Cell Tissue Kinet, 16:19-29.

Nakagawa T, Nabeshima YI, Yoshida S. 2007. Functional identification of the actual and potential stem cell compartments in mouse spermatogenesis. Dev Cell, 12:195-206.

Nakagawa T, Sharma M, Nabeshima Y, Braun RE, Yoshida S. 2010. Functional hierarchy and reversibility within the murine spermatogenic stem cell compartment. Science, 328:62-67.

Oakberg EF. 1957. Duration of spermatogenesis in the mouse. Nature, 180:1137-1138.

Oakberg EF. 1971. Spermatogonial stem-cell renewal in the mouse. Anat Rec, 169:515-531.

Oatley MJ, Kaucher AV, Racicot KE, Oatley JM 2011. Inhibitor of DNA binding 4 is expressed selectively by single spermatogonia in the male germline and regulates the self-renewal of spermatogonial stem cells in mice. Biol Reprod, 85:347356.
Russell LD, Ettlin RA, Hikim APS, Clegg ED. 1990. Histological and Histopathological Evaluation of the Testis. Clearwater, Fl: Cache River Press. 286 pp.

Sada A, Suzuki A, Suzuki H, Saga Y. 2009. The RNA-binding protein NANOS2 is required to maintain murine spermatogonial stem cells. Science, 325:13941398.

Sun F, Xu Q, Zhao D, Degui Chen C. 2015. Id4 marks spermatogonial stem cells in the mouse testis. Sci Rep, 5:17594.

Tegelenbosch RA, de Rooij DG. 1993. A quantitative study of spermatogonial multiplication and stem cell renewal in the $\mathrm{C} 3 \mathrm{H} / 101 \mathrm{~F} 1$ hybrid mouse. Mutat Res, 290:193-200.

Yang QE, Nagaoka SI, Gwost I, Hunt PA, Oatley JM. 2015. Inactivation of retinoblastoma protein (Rb1) in the oocyte: evidence that dysregulated follicle growth drives ovarian teratoma formation in mice. PLoS Genet, 11:e1005355.

Yoshida S, Sukeno M, Nabeshima YI. 2007. A vasculature-associated niche for undifferentiated spermatogonia in the mouse testis. Science 317:17221726.

Zheng K, Wu X, Kaestner KH, Wang PJ. 2009. The pluripotency factor LIN28 marks undifferentiated spermatogonia in mouse. BMC Dev Biol 9:38. doi: 10.1186/1471-213X-9-38 